\title{
Photoacoustic quantification of the optical absorption cross-sections of gold nanostructures
}

Chulhong Kim, Eun Chul Cho, Fei Zhou, Claire M. Cobley, Kwang Hyun Song, et al.

Chulhong Kim, Eun Chul Cho, Fei Zhou, Claire M. Cobley, Kwang Hyun Song, Jingyi Chen, Zhi-Yuhan Li, Younan Xia, Lihong V. Wang, "Photoacoustic quantification of the optical absorption cross-sections of gold nanostructures," Proc. SPIE 7564, Photons Plus Ultrasound: Imaging and Sensing 2010, 756430 (23 February 2010); doi: 10.1117/12.843554

SPIE. Event: SPIE BiOS, 2010, San Francisco, California, United States 


\title{
Photoacoustic quantification of the optical absorption cross-sections of gold nanostructures
}

\author{
Chulhong Kim, ${ }^{1}$ Eun Chul Cho, ${ }^{1}$ Fei Zhou, ${ }^{2}$ Claire M. Cobley, ${ }^{1}$ Kwang Hyun Song, ${ }^{3}$ Jingyi Chen, ${ }^{1}$ \\ Zhi-Yuhan Li, ${ }^{2}$ Younan Xia, ${ }^{1}$ and Lihong V. Wang ${ }^{1 *}$ \\ ${ }^{1}$ Department of Biomedical Engineering, Washington University in St. Louis, Campus Box 1097, \\ One Brookings Dr., St. Louis, Missouri, 63130 \\ ${ }^{2}$ Laboratory of Optical Physics, Institute of Physics, Chinese Academy of Sciences, Beijing 100080, \\ China \\ ${ }^{3}$ Division of Medical Physics \& Engineering, Department of Radiation Oncology, The University of \\ Texas Southwestern Medical Center at Dallas, 5323 Harry Hines Boulevard, Dallas, Texas 75390 \\ *Corresponding author: 1hwang@,biomed.wustl.edu
}

\begin{abstract}
This study demonstrates a method for measuring the optical absorption cross-sections $\left(\sigma_{a}\right)$ of Au-Ag nanocages and $\mathrm{Au}$ nanorods using photoacoustic (PA) sensing. PA signals are directly proportional to the absorption coefficient $\left(\mu_{a}\right)$ of the nanostructure. For each type of nanostructure, we first obtained $\mu_{a}$ from the PA signal by benchmarking against a linear calibration curve (PA signal vs. $\mu_{a}$ ) derived from a set of methylene blue solutions with different concentrations. We then calculated $\sigma_{a}$ by dividing the $\mu_{a}$ by the corresponding concentration of the Au nanostructure. Additionally, we obtained the extinction cross-section ( $\sigma_{e}$, sum of absorption and scattering cross-sections) from the extinction spectrum recorded using a conventional UV-vis-NIR spectrometer. From the measurements of $\sigma_{a}$ and $\sigma_{e}$, we were able to easily derive both the absorption and scattering cross-sections for each type of gold nanostructure. This method can potentially provide the optical absorption and scattering properties of gold nanostructures and other types of nanomaterials.
\end{abstract}

Keywords: Photoacoustic tomography, gold nanoparticles, optical absorption cross-sections, optical scattering crosssections.

\section{INTRODUCTION}

$\mathrm{Au}$ nanostructures have received considerable attention in biomedical applications as diagnostic contrast agents and therapeutic agents. Since the nanostructures have significant tunable properties in sizes, shapes, and compositions, their biomedical applications are broad. Specially, the localized surface plasmon resonance (LSPR) peak wavelengths of the nanostructures can be easily tuned to the visible and near-infrared (NIR) regimes by manipulating sizes and geometries. ${ }^{1-5}$ Therefore, the nanostructures have been widely applied as diagnostic contrast agents in optical imaging ${ }^{6-8}$ and therapeutic contrast agents in photothermal therapies. ${ }^{9}$ Furthermore, the nanostructures are biocompatible, and do not have potential heavy metal toxicity because they are made of gold. Despite the optical properties of nanoparticles, the molar extinction coefficients are measurable only using a spectra-photometer and Beer's law. So far, theoretical simulations have been used to estimate the absorption and scattering cross-sections of $\mathrm{Au}$ nanostructures. ${ }^{10-13}$ To maximize the advantages of the nanoparticles in biomedical applications, the absorption and scattering cross-sections need to be experimentally quantified.

Photoacoustic (PA) imaging ${ }^{7,8}$ is a hybrid imaging modality that can provide strong optical absorption contrast and high ultrasonic resolution. PA waves are generated through thermo-elastic expansion when a short-pulsed laser illuminates a target which absorbs the light. By measuring the arriving PA signals over a period of time, one-

Photons Plus Ultrasound: Imaging and Sensing 2010, edited by Alexander A. Oraevsky, Lihong V. Wang, Proc. of SPIE Vol. 7564, 756430 - (c) 2010 SPIE · CCC code: 1605-7422/10/\$18 · doi: 10.1117/12.843554 
dimensional images (A-lines) are obtained. Additional raster scanning along two transverse directions enables the formation of three-dimensional images of optical absorption heterogeneities in biological tissue.

In this study, we have experimentally evaluated the optical absorption cross-sections of $\mathrm{Au}$ nanocages, $\mathrm{Au}$ nanorods, and Au nanoshpheres using PA sensing. Since PA signals are proportional to optical absorption ${ }^{14}$, the absorption cross-sections of the three nanostructures can be quantified by comparing the PA signals of the nanostructures with those of methylene blue, which has a known absorption coefficient.

\section{METHODS AND MATERIALS}

We used four kinds of Au nanostructures: two Au nanocages, Au nanorods, and Au nanospheres. Figure 1a shows a TEM image of Au nanocages of $50.0 \mathrm{~nm}$ outer edge length, and Figure $1 \mathrm{~b}$ shows the optical absorbances of Au nanocages and methylene blue dye. Two sizes of Au nanocages (50 nm and $28 \mathrm{~nm}$ outer length) were prepared by galvanic replacement of $\mathrm{Ag}$ nanocubes with $\mathrm{AuCl}_{4}{ }^{5} \mathrm{Au}$ nanorods (44 $\times 20 \mathrm{~nm}$ in length and width, respectively) were synthesized based on the method by El-sayed et al. ${ }^{15} \mathrm{We}$ used commercially available Au nanospheres $\sim 150 \mu \mathrm{m}$ in diameter (Ted Pella Inc.). The absorption peak wavelengths were tuned to $650 \mathrm{~nm}$, which is close to the absorption peak wavelength of methylene blue. Methylene blue dye was used as a reference since its molar extinction coefficients are well known ${ }^{16}$ and it is an optical absorption dominant dye.

(a)

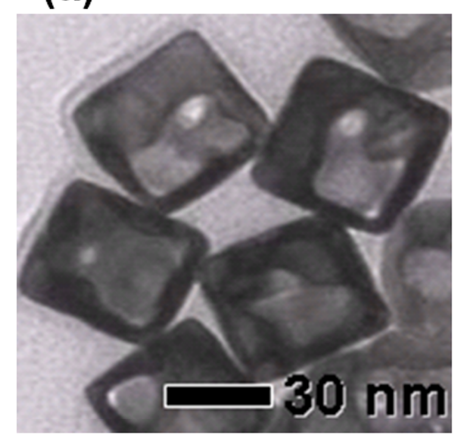

(b)

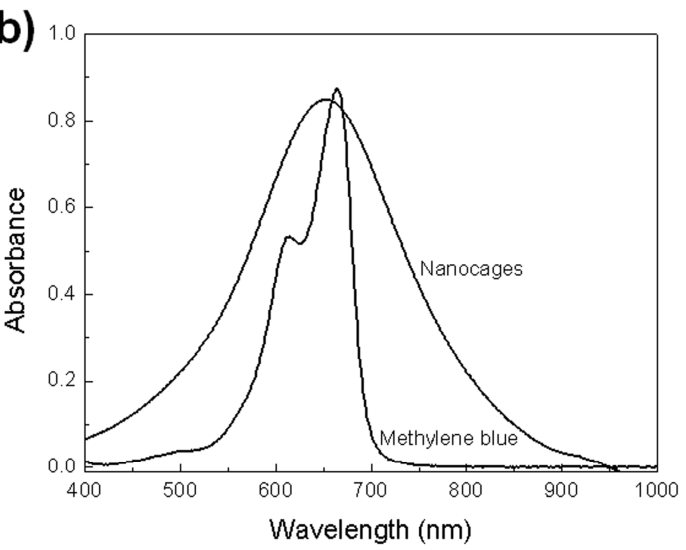

Figure 1: (a) A TEM image of Au nanocages of a $50 \mathrm{~nm}$ outer edge length and a $5.8 \mathrm{~nm}$ wall thickness. (b) UV-Vis spectra of Au nanocages and methylene blue.

Figure 2 shows an experimental schematic of the PA imaging system. A tunable dye laser (ND6000, Continuum) pumped by a Q-switched Nd:YAG laser (LS-2137, LOTIS) produced 6.5-ns laser pulses at a 10-Hz pulse repetition rate. The ring-shaped light illumination $(\lambda=638 \mathrm{~nm})$ was coaxially focused with the ultrasound focal zone in water. A single-element 5-MHz ultrasound transducer (V308, Panametrics-NDT) detected PA waves. The spatial resolutions were $138 \mu \mathrm{m}$ in the axial direction and $490 \mu \mathrm{m}$ in the transverse direction. Three Tygon ${ }^{\mathrm{R}}$ tubes were embedded inside an optically scattering medium which protected the Au nanostructures and methylene blue dye from damaging and photobleaching. Au nanostructures and dye solutions were injected into the tubes. For consistent data, each solution was ejected through the tube after each measurement, and fresh solution was refilled into the tube for additional measurements. 

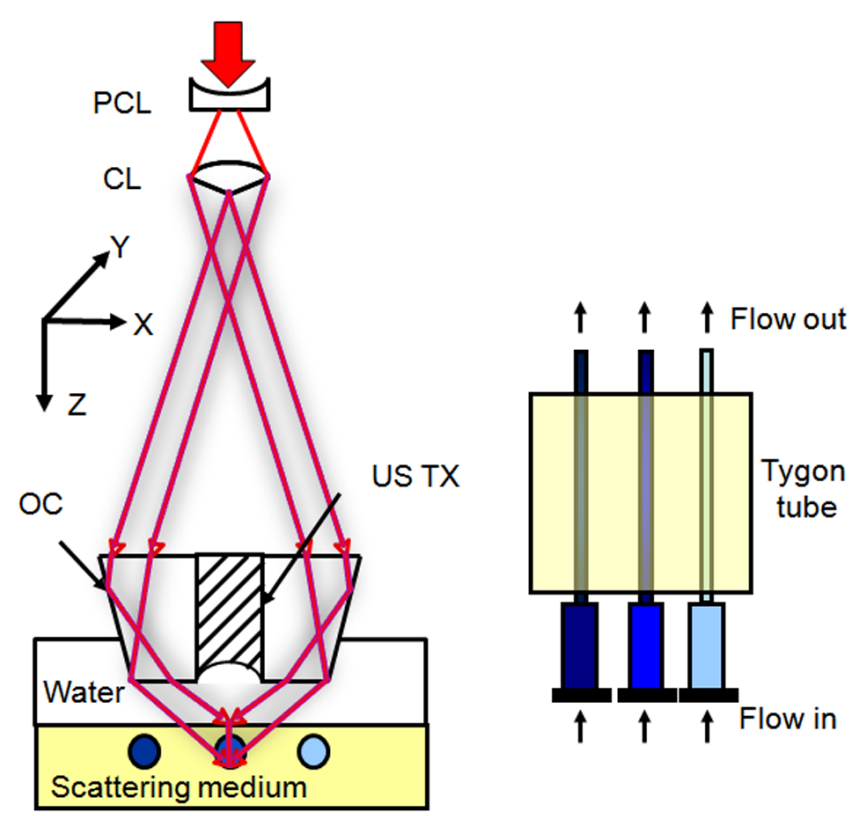

Figure 2: Experimental schematic of a photoacoustic sensing system. PCL; plano-concave lens, CL; conical lens, OC; optical condenser, US TX; ultrasound transducer.

\section{RESULTS AND DISCUSSION}

First, we checked the linear relationship between PA amplitudes and the concentration of Au nanostructures. The PA signals linearly increased with linear increments of the concentrations of the Au nanostructures $\left(\mathrm{R}^{2}=0.98 \sim 0.99\right)$. Due to the low absorption coefficients of the Au nanospheres, we could not detect any PA signal. ${ }^{12}$ Using the following equation, we could quantify the absorption coefficients of the Au nanostructures:

$$
\mu_{a, A u}=N_{A u} \sigma_{a, A u},
$$

where $\mu_{a, A u}$ is the absorption coefficient $\left(\mathrm{m}^{-1}\right), \sigma_{a, A u}$ is the absorption cross-section $\left(\mathrm{m}^{2}\right)$, and $N_{A u}$ is the number density (number of particles $/ \mathrm{m}^{3}$ ) of the Au nanostructures. Since the absorption coefficients of the Au nanostructures were unknown, we estimated them. First, we measured PA signals at various concentration of methylene blue. Since the absorption coefficients of methylene blue are well known, we estimated the relationship between PA signals and the absorption coefficients as follows $\left(\mathrm{R}^{2}=0.99\right)$ :

$$
\mu_{a}=11.99 \times \text { PA signals }-5.92 .
$$

In this approach, we assumed that optical absorption is dominant in methylene blue. To prove this, we compared the known absorption cross-section ${ }^{16}\left(2.391 \times 10^{-20} \mathrm{~m}^{2}\right)$ of methylene blue with the estimated extinction cross-section $\left(2.397 \times 10^{-20} \mathrm{~m}^{2}\right)$ from our spectra-photometer measurement. Since the two values were almost same, our assumption was valid. Then, we estimated the absorption coefficients of three Au nanostructures by using Eq. (2) and the measured PA signals in Fig. 3. Finally, we calculated the absorption cross-sections of the three nanostructures by plugging the experimentally used concentrations and the estimated absorption coefficients into Eq. (1). 


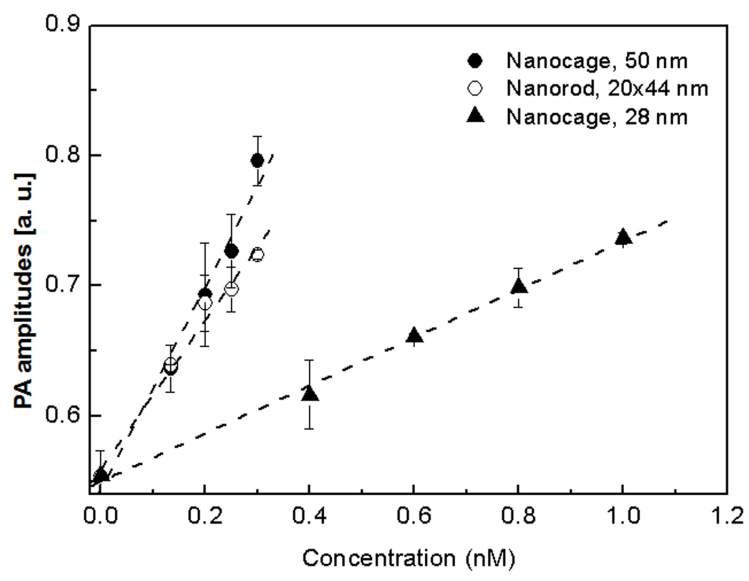

Figure 3: Plots of PA signals versus various concentrations of three Au nanostructures. PA signals for Au nanospheres were not detectable within our experimental conditions.

Table 1 summarizes the absorption and extinction cross-sections of the Au nanostructures. The extinction cross-sections were obtained from spectra-photometer measurements. The absorption peaks of Au nanostructures' spectra were tuned to $650 \mathrm{~nm}$. It is known that Au nanocages and Au nanorods are optically absorptive materials. The ratios of optical absorption cross-section to extinction cross-section of Au nanocages and Au nanorods are more than $80 \%$. Although the smaller nanocages has a higher ratio of the absorption to extinction cross-sections, the absolute absorption crosssection of the larger nanocages are wider. These results agree well with previous results ${ }^{11}$. In addition, the absorption to extinction cross-section ratio of the Au nanorods is close to the ratio from the larger Au nanocages. Moreover, since the absorption cross-section of the Au nanospheres was calculated to be only $5 \%$ of the extinction, it was difficult to detect PA signals experimentally.

Table 1: Experimental optical extinction and absorption cross-sections of Au nanostructures. All the values were obtained at $638 \mathrm{~nm}$. PA signals were not detected from Au nanospheres.

\begin{tabular}{cccc}
\hline & \multicolumn{2}{c}{ Cross-section $\left(10^{-15} \mathrm{~m}^{-2}\right)$} & $\begin{array}{c}\text { Absorption/ } \\
\text { Extinction }\end{array}$ \\
\cline { 2 - 3 } & Extinction & Absorption & \\
\hline Nanocage, $50 \mathrm{~nm}$ & $2.38 \pm 0.02$ & $2.0 \pm 0.11$ & 0.84 \\
\hline Nanocage, $28 \mathrm{~nm}$ & $0.49 \pm 0.02$ & $0.52 \pm 0.04$ & 1 \\
\hline Nanorod, 20 $\times 44 \mathrm{~nm}$ & $2.16 \pm 0.21$ & $1.81 \pm 0.29$ & 0.84 \\
\hline Nanosphere, $150 \mathrm{~nm}$ & $145 \pm 14.76$ & - & - \\
\hline
\end{tabular}

\section{CONCLUSIONS}

In summary, we have demonstrated a method to evaluate the absorption cross-sections of Au nanocages and Au nanorods using photoacoustic imaging. By comparing PA signals from Au nanostructures with those from methylene blue, which has a known absorption coefficient, we can estimate the absorption cross-sections of those nanoparticles experimentally. Based on the experimental results, we confirmed that optical absorption is dominant in Au nanocages (84 $\sim 100 \%$ of extinction) and nanorods (84\%). Therefore, we conclude that these two nanostructures can be used as 
optical absorption contrast agents for diagnostic purposes and photothermal therapeutic agents. Potentially, this method can be used to measure the optical properties of other materials, and can guide the choice of optical materials for specific applications.

\section{ACKNOWLEDGEMENT}

This work was supported in part by grants from National Institutes of Health (R01 EB000712, R01 EB008085, R01 NS46214, and U54 CA136398 -- the Network for Translational Research -- to L.V.W.). L.V.W. has a financial interest in Endra, Inc., which, however, did not support this work. Y.X. thanks the National Institutes of Health for a 2006 Director's Pioneer Award (DP1 OD000798-04). Z.Y.L. was supported by the National Natural Science Foundation of China (60736041 and 10874238). E.C.C. was also partially supported by a fellowship award from the Korea Research Foundation (KRF-2007-357-D00070) funded by the Korean Government.

\section{REFERENCES}

[1] Y. Xia and N. J. Halas, "Shape-Controlled Synthesis and Surface Plasmonic Properties of Metallic Nanostructures," MRS Bull. 30, 338-348 (2005).

[2] M. A. El-Sayed, "Some interesting properties of metals confined in time and nanometer space of different shapes," Acc. Chem. Res. 34, 257-264 (2001).

[3] M. B. Mohamed, V. Volkov, S. Link, M. A. El-Sayed, "The lightning gold nanorods: fluorescence enhancement of over a million compared to the gold metal," Chem. Phys. Lett. 317, 517 (2000).

[4] B. Wiley, Y. Sun and Y. Xia, "Synthesis of silver nanostructures with controlled shapes and properties," Acc. Chem. Res. 40, 1067-1076 (2007).

[5] S. E. Skrabalak, L. Au, X. Li and Y. Xia, "Facile synthesis of Ag nanocubes and Au nanocages," Nature Prot. 2, 2182-2190 (2007).

[6] K. J. Lee, P.D. Nallathamby, L. M. Browning, C. J. Osgood and X.-H. N. Xu, "In Vivo Imaging of Transport and Biocompatibility of Single Nanoparticles in Early Development of Zebrafish Embryos", ACS Nano, 1, 133-143, (2007).

[7] K. H. Song, C. Kim, C. M. Cobley, et al., "Near-Infrared Gold Nanocages as a New Class of Tracers for Photoacoustic Sentinel Lymph Node Mapping on a Rat Model," Nano Lett. 9(1), 183-188 (2009).

[8] K. Kim, S.-W. Huang, S. Ashkenazi, M. O'Donnell, A. Agarwal, N. A. Kotov, M. F. Denny, and M. J. Kaplan, "Photoacoustic imaging of early inflammatory response using gold nanorods", Applied Physics Letters, 90(22), 223901 (2007).

[9] D. C. Adler, S.-W.Huang, R. Huber and J.G. Fujimoto, "Photothermal detection of gold nanoparticles using phasesensitive optical coherence tomography," Opt. Exp. 16(7), 4376-4393 (2008).

[10] M. Hu, H.Petrova, A. R. Sekkinen, J. Chen, J. M. McLellan, Z.-Y. Li, M. Marquez, X. Li, Y. Xia and G.V. Hartland, "Optical Properties of Au-Ag Nanoboxes Studied by Single Nanoparticle Spectroscopy," J. Phys. Chem. B, 110, 19923-19928 (2006).

[11] J. Chen, B, Wiley, Z.-Y. Li, D. Campbell, F. Saeki, H. Cang, L. Au, J. Lee, X. Li and Y. Xia, "Gold nanocages: Engineering their structure for biomedical applications," Adv. Mater. 17, 2255-2261 (2005).

[12] J. Yguerabide and E. E. Yguerabide, "Light-scattering submicroscopic particles as highly fluorescent analogs and their use as tracer labels in clinical and biological applications," Anal. Biochem. 262, 157-176 (1998).

[13] K. L. Kelly, E. Coronado, L. Zhao, and G. C. Schatz, "The optical properties of metal nanoparticles: the influence of size, shape, and dielectric environment," J. Phys. Chem. B 107, 668-677 (2003).

[14] H. F. Zhang, K. Maslov, G. Stoica, et al., "Functional photoacoustic microscopy for high-resolution and noninvasive in vivo imaging," Nat. Biotechnol. 24(7), 848-851 (2006).

[15] B. Nikoobakht, and M. A. El-Sayed, "Preparation and growth mechanism of gold nanorods (NRs) using seedmediated growth method," Chem. Mater. 15, 1957-1962 (2003).

[16] http://omlc.ogi.edu/spectra/mb/index.html 